\title{
Improving national surveillance of new HIV diagnoses
}

\author{
N Popovic ${ }^{1 *}$, Q Yang ${ }^{1}$, N Haddad ${ }^{1}$, A Weeks ${ }^{1}$, C Archibald ${ }^{1}$
}

\begin{abstract}
The purpose of national HIV surveillance is to track and summarize trends in newly diagnosed cases as an indicator of HIV transmission within Canada, and supports the development and evaluation of programs and policies for prevention, testing and delivery of care. Accurately capturing and interpreting trends in HIV diagnoses within national surveillance becomes complicated when there is movement of people within a country or when individuals are diagnosed with HIV prior to migrating to a new country. This has been identified as an issue in other countries, including Australia, New Zealand and Switzerland. The Public Health Agency of Canada (PHAC) recently assessed this in Canada after noting a rise in new HIV cases in Canada between 2014 to 2017. An environmental scan was conducted to better understand how new and previously diagnosed cases of HIV were recorded by and reported to PHAC from provincial and territorial (PT) public health authorities. It was discovered there was variation with respect to the reporting of cases who had received a new diagnosis of HIV within the province or territory, but who had previously received an HIV diagnosis from another PT or another country. Five PTs included cases previously diagnosed in another Canadian PT within the HIV surveillance data reported to PHAC and nine PTs included people who were diagnosed with HIV outside of Canada. The provincial and territorial public health authorities then reviewed HIV surveillance data from 2007 to 2017 to identify cases using a common definition of "previous HIV-positive test result". This included any case who gave a history, or had laboratory evidence, of an HIV-positive result from another PT or another country before presenting for care in the province or territory where they now resided. When these cases were subtracted from the total, a revised number of new HIV diagnoses was calculated for Canada. Re-analysis of surveillance data using this common definition for 2007 to 2017 explained more than half of the increase in HIV cases that had been documented in Canada over the last four years. In the future, national surveillance data will be calculated adopting this new common definition of a previous positive test result, in order to more accurately describe the trends in HIV transmission occurring in Canada.
\end{abstract}

This work is licensed under a Creative Commons Attribution 4.0 International License.

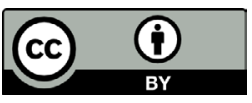

Affiliation

${ }^{1}$ Centre for Communicable Diseases and Infection Control, Public Health Agency of Canada, Ottawa, ON

*Correspondence: nashira. popovic@canada.ca

Suggested citation: Popovic N, Yang Q, Haddad N, Weeks A, Archibald C. Improving national surveillance of new HIV diagnoses. Can Commun Dis Rep 2019;45(12):313-6. https://doi.org/10.14745/ccdr.v45i12a02

Keywords: HIV, AIDS, epidemiology, surveillance

\section{Introduction}

In Canada, national HIV surveillance is conducted by the Public Health Agency of Canada (PHAC) through the annual voluntary submission of data on new HIV diagnoses by all Canadian provinces and territories (PTs) (1). The purpose of national HIV surveillance is to track and summarize trends in newly diagnosed cases as an indicator of HIV transmission within Canada, which supports the development and evaluation of programs and policies for prevention, testing, and delivery of care. Within national HIV surveillance, a new HIV diagnosis is defined as a person's first HIV positive test result in Canada. However, variations existed regarding how people previously diagnosed with HIV in other PTs or other countries were reported by PTs, especially when there was no laboratory report available.
Depending on whether these cases are included or not can affect the trends in new HIV diagnoses.

The challenge in counting people who have received a diagnosis of HIV and then move to a new place and received care in that area, has been dealt with in different ways internationally. For example, both Australia and New Zealand collect information on where a person was first diagnosed with HIV (i.e. in the country versus overseas), but report this information in different ways in their respective surveillance reports $(2,3)$. New Zealand includes previous positive overseas diagnoses in their annual HIV case counts, whereas Australia excludes them and presents them separately, to provide a more accurate representation of the 
transmission occurring within the country. In contrast, Switzerland reports on the HIV status of foreign nationals now living in the country, but does not provide any distinction as to whether or not the person was diagnosed prior to arrival in the country (4). The European Centre for Disease Prevention and Control includes a separate section within its surveillance report on the probable region of infection; however, these cases are included in overall surveillance counts (5).

In Canada, the number of reported HIV cases increased by 16\% from 2014 to 2017 (1). There are a number of possible explanations for this increase, including increased testing, increased HIV transmission (1), and possible variations in reporting practices such as the inclusion of HIV cases moving into a new province or territory and the number of HIV-positive people migrating to Canada. For example, a person who has his/her first HIV diagnosis in province $A$ is reported to PHAC as a new HIV diagnosis. If this person then moves to province $B$ and is retested for HIV when they enter care, this test result may again be reported to $\mathrm{PHAC}$ as a new diagnosis by province $B$, if province $B$ is not aware or does not report to PHAC that this person was previously diagnosed elsewhere. The PTs do not report personal identifiers, so PHAC is unable to delete duplicate case reports at the national level. In addition, PTs may have information on people migrating to Canada who have been previously diagnosed with HIV in another country, either through the person reporting his/her previous diagnosis to their health care provider or through HIV testing as part of the Immigration Medical Exam conducted by Immigration, Refugees and Citizenship Canada. Persons who have been previously diagnosed with HIV in another country could be reported to PHAC's national surveillance as a new HIV diagnosis if the health care provider is not aware that the person was previously diagnosed with HIV or if the health care provider does not report this information to public health.

This article describes an initial analysis completed by PHAC in collaboration with all the PTs, to evaluate the impact on the national trend of new HIV diagnoses in Canada when a common definition and methodology is used for previously diagnosed cases of HIV.

\section{Analysis}

Between December 2018 and April 2019, an environmental scan was conducted with all technical laboratory and epidemiological partners from Canadian PT public health authorities to better understand how previously diagnosed cases of HIV have been recorded in each PT and reported to PHAC. Results from this environmental scan showed that five PTs included cases previously diagnosed in another Canadian PT within the HIV surveillance data that they reported to PHAC and nine PTs included people who were diagnosed with HIV outside of Canada. The PT public health authorities were then asked to review the annual surveillance counts that they provided to PHAC between 2007 and 2017, and to identify the number of cases with a known previous HIV-positive test result (either through laboratory evidence or self-reported) from another PT or from another country ("previous positives"). The methods used for reviewing historical surveillance data varied by PT, depending on their respective surveillance systems, and included a review of a specific field or code within an electronic case management system or a review of paper-based forms.

As a result of this review, revised counts of new HIV diagnoses for the years 2007 to 2017 were calculated, with individuals with a previous HIV-positive test result from another Canadian PT removed to reduce double-counting at the national level. In addition, individuals with a known HIV-positive test result from outside of Canada were removed to better assess trends in HIV transmission within Canada.

Trends in the national number of new HIV diagnoses for 2007 to 2017 are shown in Figure 1. The solid line displays the trend in the reported number of HIV diagnoses as presented in PHAC's annual surveillance reports, based on historical PT reporting practices (1). The dotted line shows the revised trend in HIV diagnoses following the removal of known previous positives. For most years, the revised trend follows the same pattern as the currently reported trend, with an average of 200 fewer HIV diagnoses each year. This pattern is continued until 2017 when the revised number of HIV diagnoses does not continue to increase like the original surveillance number. When previous positives were identified and removed, the percent change from 2014 to 2017 went from a $16 \%$ increase down to a $9 \%$ increase.

\section{Figure 1: Number of new HIV diagnoses in Canada 2007-2017 based on historical methodology compared to the new common definition of "previous positives"}

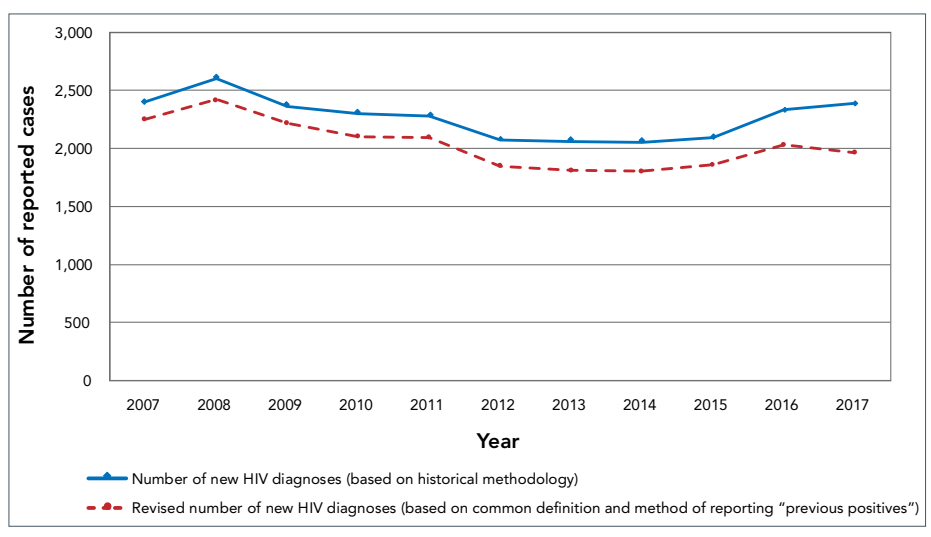

\section{Discussion}

In an effort to reduce double-counting and to more accurately represent trends in HIV transmission occurring within Canada, PHAC worked with the PTs to identify reported cases of HIV that had evidence of a previous positive HIV diagnosis from outside the PT or country. A reanalysis of HIV data between 2014-2017 using a common definition and method showed that instead of a $16 \%$ increase in newly reported cases of HIV there was a $9 \%$ 
increase. This indicates that previously diagnosed cases of HIV accounts for a little more than half of the increase in HIV cases in Canada reported to PHAC by the PTs over the past four years. Additionally, in 2017 when the historical methodology was used, there was a slight increase in new diagnoses compared with 2016. However, when the common definition and method was used, it resulted in a slight decrease in 2017 compared with 2016. This suggests that in 2017 the reported increase in new diagnoses was a result of an increase in previously diagnosed HIV cases, rather than an increase in HIV transmission in Canada or other factors.

Ontario has undertaken similar work at the provincial level and has also found that the increase in the number of new HIV diagnoses in recent years was largely due to "out-of-province" previous diagnoses $(6,7)$. In addition, several provinces already separate previously diagnosed cases of HIV from their annual number of new HIV diagnoses within provincial surveillance reports (8-10). Globally, the various approaches used to account for known previous positives in national HIV surveillance systems demonstrate the complexity of this issue and highlight the differences in the definitions of previous positive and in reporting practices (2-5).

Several limitations to this analysis should be considered when interpreting these results. First, the number of previous positives could be an underestimate, since it often relies on self-disclosure of a previous HIV diagnosis. In addition, persons who were first tested anonymously and later diagnosed nominally may produce duplicate positives that cannot be identified and removed. Lastly, PHAC's ability to detect previously diagnosed cases is limited as there are not enough identifiers to detect duplicates.

\section{Next steps}

Tracking and reporting on HIV cases is a complex and decentralized process. Going forward, PHAC will monitor both:

- The total number of reported HIV diagnoses in Canada (consistent with previous surveillance reports) to assess the burden of HIV in the country

- The number of new HIV diagnoses (not known to be previously diagnosed) to inform trends in HIV transmission occurring within Canada

Migrants who have been diagnosed with HIV outside of Canada will continue to be included in PHAC's national estimates of HIV prevalence to provide an accurate national picture of the number of people living with HIV who require care. PHAC will also work with PTs to collect additional demographic information on previously diagnosed cases of HIV from outside of Canada to help inform public health prevention and control efforts for this group.

\section{Conclusion}

PHAC will continue to work with PT public health authorities to standardize reporting of previously diagnosed individuals who have recently moved. The use of a common approach for reporting previous HIV diagnoses explains more than half of the increase in HIV cases over the last four years. Future national surveillance reports will include data based on this revised definition/methodology to more accurately describe the trends in HIV transmission occurring within Canada.

\section{Authors' statement}

This work was completed by employees of the Public Health Agency of Canada. Authors' contributions are outlined below: NP - Conceptualization, data curation, interpretation of data, writing original draft, review, editing, validation, writing final draft, visualization

QY - Interpretation of data, contributed to first draft

$\mathrm{NH}$ - Contributed to first draft

AW - Contributed to first draft

CA - Conceptualization, review/revision of the paper, final approval

\section{Conflict of interest}

None.

\section{Acknowledgements}

The Public Health Agency of Canada would like to acknowledge all provincial and territorial HIV/AIDS programs for their contribution and participation.

\section{Funding}

This work was supported by the Public Health Agency of Canada as part of its core mandate.

\section{References}

1. Haddad N, Li JS, Totten S, McGuire M. HIV in Canada-Surveillance Report, 2017. Can Commun Dis Rep 2018;44(12):348-56. DOl PubMed

2. The Kirby Institute. University of New South Wales. HIV in Australia Annual surveillance short report 2018. Sydney (Australia); Kirby Institute UNSW: 2018. https://kirby.unsw. edu.au/sites/default/files/kirby/report/supplHIV2018_ content_20180920r.pdf

3. New Zealand AIDS Foundation. HIV in New Zealand. https:// www.nzaf.org.nz/hiv-aids-stis/hiv-aids/hiv-in-new-zealand/

4. Swiss Federal Office of Public Health. Statistiques et analyses concernant VIH/IST. Berne (Switzerland); OFSP: modified 2019. https://www.bag.admin.ch/bag/fr/home/ zahlen-und-statistiken/zahlen-zu-infektionskrankheiten/ hiv-sti-statistiken-analysen-trends.html\#accordi on_13805488611549284685179 
5. European Centre for Disease Prevention and Control and the World Health Organization Regional Office for Europe. HIV/ AIDS Surveillance in Europe, 2018. Copenhagen (Denmark); WHO: 2018. https://ecdc.europa.eu/sites/portal/files/ documents/hiv-aids-surveillance-europe-2018.pdf

6. Ontario HIV Epidemiology and Surveillance Initiative. Public Health Ontario. Refining HIV surveillance on new HIV diagnoses in Ontario. Toronto (ON); OHESI: 2018. http://www.ohesi.ca/refining-hiv-surveillance-o n-new-hiv-diagnoses-in-ontario/

7. Ontario HIV Epidemiology and Surveillance Initiative. Public Health Ontario. New HIV diagnoses in Ontario, 2017. Toronto (ON); OHESI; 2019. http://www.ohesi.ca/ wp-content/uploads/2019/07/OHESI-2017-new-diagnoses. pdf
8. Government of New Brunswick. New Brunswick Communicable Disease 2017 Annual Surveillance Report. Government of NB: 2018. https://www2.gnb.ca/content/ dam/gnb/Departments/h-s/pdf/en/CDC/HealthProfessionals/ Annual_Report_CDC_Branch_2017.pdf

9. Ministry of Health. Population Branch. Government of Saskatchewan. HIV Prevention and Control Report 2017. http://publications.saskatchewan.ca/\#/products/91148

10. Health M. Seniors and Active Living. 2017 Annual Statistical Update: HIV in Manitoba. Manitoba Health: 2018. https:// www.gov.mb.ca/health/publichealth/surveillance/hivaids/ docs/dec2017.pdf 